\title{
O Apoio Matricial na qualificação da Atenção Primária à Saúde às pessoas com doenças crônicas
}

\author{
Matrix Support in the qualification of the Primary Health Care for \\ people with chronic diseases
}

Cássia Regina Gotler Medeiros ${ }^{\mathbf{1}}$, Ana Luísa Freitag1, Luísa Scheer Ely Martines'1, Olinda Maria de Fátima Lechmann Saldanha', Magali Quevedo Grave1, Lydia Koetz Jager', Gisele Dhein $\mathbf{1}$

DOI: $10.1590 / 0103-1104202012515$

RESUMO Objetivou-se analisar o potencial do dispositivo de Apoio Matricial na qualificação da Atenção Primária à Saúde das pessoas com Doenças Crônicas Não Transmissíveis (DCNT). Trata-se de pesquisa-intervenção com equipes de saúde da família em dois municípios do Rio Grande do Sul, aplicando a estratégia de Apoio Matricial. Realizaram-se Grupos Focais para avaliar as atividades. Na Análise de Conteúdo, emergiram duas categorias: 'Dificuldades na gestão e no cuidado às pessoas com DCNT' e 'Qualificação do cuidado às pessoas com DCNT após o Apoio Matricial'. Reuniões sistemáticas foram implementadas após os apoios, reconhecendo-as com potencial para qualificar as práticas. O Apoio Matricial promoveu a comunicação entre os profissionais e a aproximação entre os membros da equipe, mostrando-se efetivo no empoderamento das equipes de Atenção Primária à Saúde, impulsionando mudanças nas práticas de gestão e cuidado às pessoas com DCNT.

PALAVRAS-CHAVE Atenção Primária à Saúde. Doença crônica. Gestão em Saúde. Educação em Saúde.

\begin{abstract}
The aim of this study was to analyze the potential of Matrix Support devices in qualifying Primary Health Care for people with Chronic Noncommunicable Diseases (CNCD). It is a research intervention with Family Health Strategy teams in two municipalities of Rio Grande do Sul, applying the Matrix Support strategy. Focal Groups were used to measure the activities. In Content Analysis, two categories emerged: 'Difficulties in the management and care of people with CNCD' and 'Qualification of care for people with CNCD after Matrix Support'. Systematic meetings with the entire team were implemented, recognizing them with the potential to qualify the practices from their problematization. Matrix Support promoted the communication between professionals and the approximation of the other team members, being effective in the empowerment of Primary Health Care teams, boosting changes in management practices and care for people with CNCD.
\end{abstract}

KEYWORDS Primary Health Care. Chronic disease. Health management. Health education. 


\section{Introdução}

A transição demográfica acelerada reflete no envelhecimento da população e na incidência das Doenças Crônicas Não Transmissíveis (DCNT). Estas doenças foram causa de aproximadamente $70 \%$ da mortalidade mundial em 2015, com prevalência das doenças cardiovasculares'. Em 1990, a mortalidade proporcional por DCNT no Brasil correspondia a 59,6\% das mortes, aumentando em 2015 para $75,8 \%{ }^{2}$.

A Portaria $\mathrm{n}^{\circ} 252$ instituiu a Rede de Atenção à Saúde (RAS) das pessoas com DCNT no âmbito do Sistema Único de Saúde (SUS) ${ }^{3}$. Essa Portaria propõe mudanças no modelo de atenção, ampliando as ações de promoção da saúde e prevenção de complicações das DCNT. Em 2014, a Portaria ${ }^{\circ}$ 483 redefiniu a RAS das pessoas com DCNT e estabeleceu diretrizes para a organização das suas linhas de cuidado ${ }^{4}$.

Estudo realizado em municípios da $16^{\mathrm{a}}$ Coordenadoria Regional de Saúde (16 $\left.{ }^{\mathrm{a}} \mathrm{CRS}\right)$, no Rio Grande do Sul (RS), verificou que as internações e óbitos evitáveis diminuíram com o aumento da cobertura da Estratégia Saúde da Família (ESF). No entanto, a análise das trajetórias assistenciais de usuários sugeriu baixa efetividade da Atenção Primária à Saúde (APS) tanto na prevenção quanto no diagnóstico das DCNT. Observou-se que a busca por cuidados em saúde, muitas vezes, inicia-se nos serviços de média e alta densidade tecnológica, sendo a APS acessada posteriormente ao diagnóstico das DCNT em outros pontos da rede ${ }^{5}$.

Uma das estratégias para qualificação da APS é a problematização das práticas e a promoção da cogestão do cuidado, por meio do Apoio Matricial (AM). O AM teve início na década de 1990 no Brasil, sendo implementado por profissionais da APS e da saúde mental em Campinas, no estado de São Paulo. Os seus resultados nesse cenário contribuíram para que algumas políticas do Ministério da Saúde (MS) - Humaniza-SUS, saúde mental e ESF - incorporassem essa perspectiva ${ }^{6}$. Ele visa ao suporte e à corresponsabilização dos diferentes atores pelas demandas de saúde da comunidade. Refere-se a uma estratégia de partilha dos processos de trabalho entre a equipe da APS e a equipe especializada, que propicia sua retaguarda quando necessário ${ }^{7}$. $\mathrm{O}$ apoiador matricial é um especialista com saberes e perfil diferentes da equipe de referência, a qual é responsável pelo acompanhamento longitudinal do usuário, agregando recursos que aumentam a resolutividade das intervenções ao usuário. Espaços dialógicos são construídos, a fim de elaborar um projeto terapêutico integrado. As relações entre apoiador e equipe de referência devem ser horizontais, possibilitando a criação compartilhada da solução para os problemas clínicos ${ }^{6}$.

O momento é oportuno para a implementação de tecnologias inovadoras de gestão e para a organização de redes de cuidado às pessoas com $\mathrm{DCNT}^{8}$. Ao analisar o estado da arte referente ao AM, observou-se que a implementação dessa estratégia se concentra, em sua maioria, no campo da saúde mental, não se encontrando estudos na área das DCNT ${ }^{9,10}$. Considerando que estas constam como uma das metas prioritárias de pesquisa na agenda do MS, em reconhecimento ao impacto social e econômico que representam para o País e sua condição sensível à APS, melhorar a assistência pode significar mais qualidade de vida aos usuários e um sistema de saúde mais competente e resolutivo ${ }^{11}$.

Em vista do contexto regional e das necessidades do atual perfil de saúde no Brasil, este estudo objetivou analisar o potencial do dispositivo de AM na qualificação da APS às pessoas com DCNT.

\section{Material e métodos}

Implementou-se o estudo em dois municípios (doravante denominados A e B) da Região de Saúde 29 do Rio Grande do Sul, selecionados por manifestação de interesse dos respectivos gestores, após divulgação em uma reunião da Comissão Intergestores Regional. Essa Região 
é composta por 37 municípios e está sob a abrangência administrativa da $16^{\mathrm{a}} \mathrm{CRS}$.

Constituiu-se em uma pesquisa-intervenção, com abordagem qualitativa e utilização de métodos participativos, como o da roda e grupo focal12,13. Ocorreram 14 encontros mensais de AM em cada um dos municípios, nos anos de 2015 e 2016, conduzidos por duas equipes interprofissionais de pesquisadores e estudantes de uma Instituição de Ensino Superior do RS. Participaram 40 trabalhadores da saúde (duas equipes de ESF do município A e uma do município B) - Agentes Comunitários de Saúde (ACS), enfermeiras, técnicas de enfermagem, fisioterapeutas, farmacêuticos, nutricionistas, dentistas, auxiliares de saúde bucal, médicos e psicólogas. Os gestores optaram por não participar, de modo a não interferir no posicionamento das equipes.

As equipes de ESF participaram da intervenção separadamente, em uma sala disponibilizada pelas unidades de saúde em cada município. O processo de AM ocorreu por meio de diversos recursos dinâmicos e interprofissionais. Os temas debatidos foram pactuados a partir das demandas das equipes. Realizou-se o AM com base na discussão da situação de saúde das comunidades, projetos terapêuticos singulares e educação permanente. As demandas se voltaram, também, para as dificuldades da gestão, do planejamento e avaliação em saúde, bem como das relações interprofissionais.

Os pesquisadores e estudantes iniciaram as atividades trabalhando com o entendimento que os profissionais possuíam acerca dos fluxos dos usuários com DCNT na RAS, mediante discussão de casos. Essa etapa possibilitou às equipes de saúde analisar os processos de trabalho e o cuidado oferecido às pessoas com DCNT, buscando implementar novas estratégias e aprimorar as existentes.

Ao final de 2015 e 2016, realizaram-se dois Grupos Focais (GF), um em cada município, por pesquisadores que não participaram dos encontros, permitindo a avaliação das ações de apoio e os impactos nos processos de trabalho.
Os debates duraram, em média, duas horas, sendo gravados e transcritos. As questões norteadoras desse processo de avaliação foram: 1) como os participantes avaliam o processo de AM; 2) quais os entraves e potencialidades deste processo; 3) como avaliam e identificam em suas práticas os efeitos do AM.

Neste artigo, apresentam-se os resultados da análise dos relatos dos GF, em que foram identificadas unidades de significado que constituíram categorias de análise, conforme o método de Análise de Conteúdo ${ }^{14(37)}$, que se trata de "um conjunto de técnicas de análise das comunicações", consistindo em não perder a heterogeneidade de seu objeto, a qual é dividida em três etapas. A primeira é a pré-análise, caracterizada pela organização, envolvendo a escolha dos documentos a serem analisados, a formulação de hipóteses e objetivos, e ainda a "elaboração de indicadores que fundamentam a interpretação final"14(125). No caso desta pesquisa, o processo de pré-análise deu-se a partir da transcrição dos relatos gravados nos GF.

A segunda etapa é a exploração do material, que se define como a fase 'longa e fastidiosa', pois abrange o processo de escolha das unidades de significados, que são tomadas como base para recorte, enumeração e categorização das falas nas transcrições de cada encontro ${ }^{\mathbf{1 4}}$. A análise das categorias foi realizada a partir dos referenciais que fundamentam as políticas públicas de saúde no Brasil e orientam a Política Nacional da Atenção Básica (PNAB) e a de Educação Permanente em Saúde (EPS) ${ }^{\mathbf{1 5}, 16}$.

A última fase remete ao tratamento dos resultados e análise a partir das transcrições dos encontros. Durante essa etapa, o pesquisador reflete sobre os dados obtidos, considerando probabilidades de comparação entre categorias, retomando quantas vezes forem necessárias o referencial teórico utilizado para defini-las ${ }^{\mathbf{1 4}}$. Nesse caso, as categorias emergentes foram: 'Dificuldades na gestão e no cuidado às pessoas com DCNT' e 'Qualificação do cuidado às pessoas com DCNT após o AM'. A análise do potencial do AM para a qualificação da APS não teve foco em desfechos 
clínicos ou epidemiológicos, mas, sim, nos processos de trabalho, segundo as percepções dos profissionais envolvidos com o cuidado de pessoas com DCNT.

Respeitaram-se os aspectos éticos em conformidade com a Resolução $n^{0} 466$, do Conselho Nacional de Saúde; e o estudo foi aprovado pelo Comitê de Ética em Pesquisa da Universidade do Vale do Taquari (Univates), sob número 1.432.1217. Para garantir o sigilo, identificaram-se as falas dos participantes com a primeira letra da profissão e o município em que trabalha (A ou B).

\section{Resultados e discussão}

\section{Estrutura dos municípios participantes do estudo}

Para o atendimento às pessoas com DCNT, o município A conta com um hospital de pequeno porte e duas ESF, contemplando $100 \%$ de cobertura. Nas equipes de ESF, atuam três médicos em clínica geral, pediatra, ginecologista, duas enfermeiras, nutricionista, educador físico, psicóloga, três técnicos e dois auxiliares de enfermagem, dez ACS e três auxiliares administrativos. Há ainda um serviço de fisioterapia terceirizado. São realizadas consultas, grupos de educação em saúde e atendimento domiciliar pela equipe interprofissional, tanto na área urbana quanto na rural, semanalmente. Há rotatividade de gestores de saúde no município: o penúltimo permaneceu por dois anos, e o último, por somente cinco meses.

A população do município B é atendida na Unidade Básica de Saúde (UBS), contando com duas equipes de ESF, constituídas por quatro médicos de clínica geral, ginecologista, pediatra, psiquiatra, dois psicólogos, fisioterapeuta, cirurgião-dentista, quatro enfermeiras, nutricionista, cinco técnicos de enfermagem, seis ACS e sete auxiliares administrativos, oferecendo $100 \%$ de cobertura para população e uma unidade com leitos para observação 24 horas. Somente a equipe de uma das ESF participou do estudo. Tem, ainda, uma clínica geriátrica, que atende pessoas em cuidados paliativos. A demanda de urgência e emergência é encaminhada para o serviço de referência regional em outro município.

Em ambos, a demanda por APS é atendida no próprio município, mas a média e a alta complexidade são encaminhadas para serviços de referência regionais, ou, em poucos casos, para Porto Alegre (RS), considerando que são municípios com menos de 10 mil habitantes.

\section{Dificuldades na gestão e no cuidado às pessoas com DCNT}

Para analisar o potencial da estratégia de AM em qualificar a APS às pessoas com DCNT, foi importante conhecer o contexto das equipes municipais de saúde, que revelou as dificuldades das equipes na gestão e no cuidado dessas pessoas. Relatos dos profissionais do município B sobre o excesso de demanda permearam todo o processo de apoio e foram reforçados no GF, ressaltando a forte interferência do gestor na tomada de decisão em relação à atenção aos usuários. Em estudo realizado em São Paulo com profissionais da atenção básica, também se verificou que o atendimento emergencial e mecanizado ainda é um desafio para a APS, que enfrentam uma sobrecarga de demanda ${ }^{18}$.

Eles [gestores] também têm que parar e pensar, e a prevenção? [...] Estamos só tapando os incêndios e é demanda, demanda... (MB).

A referida exigência da gestão para que se atenda todos os usuários que procuram a ESF é colocada como impeditivo da realização de outras atividades, como reunião de equipe e promoção da saúde. Estudo realizado em Sapucaia do Sul (RS) revelou que, devido à demanda excessiva, os profissionais apenas renovavam as receitas, sem questionar o prosseguimento e as dificuldades no tratamento ${ }^{19}$. Da mesma forma, em outros países, como os 
Estados Unidos, o crescimento espantoso das DCNT, devido ao envelhecimento da população e à maior longevidade, sobrecarregou os sistemas de saúde e revelou deficiências na organização e prestação de cuidados a pacientes com essas doenças ${ }^{20}$.

Falhas na gestão dos processos de trabalho refletem na falta de planejamento conjunto das ações, reforçando um modelo assistencial predominantemente biomédico ${ }^{18}$. Uma das diretrizes da ESF é definir o território adstrito, reorganizando e procurando diminuir a demanda espontânea, a partir do momento em que os problemas de saúde vão sendo identificados e uma abordagem coletiva é colocada em prática, com ações de promoção, prevenção e atenção à saúde 21 . Contudo, neste estudo, foram relatadas algumas tentativas frustradas em relação à reorganização dos fluxos e processos de trabalho na ESF.

[...] é que desde o início a gente tentou agendar, mas como paciente não pode ser mandado embora, aí era o agendamento, mais todo o volume de atendimento, porque tem uma demanda espontânea que é difícil de conter. (MB).

Diante dessa situação, os profissionais manifestaram a preocupação com a resolutividade do atendimento, entendendo que a demanda excessiva compromete a qualidade do trabalho e impacta negativamente nos resultados da atenção às pessoas com DCNT.

[...] a gente não consegue dar suporte para isso tudo e a qualidade do nosso atendimento também cai, ... tu precisas conversar, examinar os pacientes, e não a coisa ser corrida porque tu tens que resolver demanda. (MB).

Entende-se que a inserção da promoção da saúde na prática profissional ainda é um desafio a ser vencido, uma vez que, apesar de reconhecer sua importância, ainda se mantém uma atenção à saúde de caráter curativo, individual e fragmentado. Na literatura, autores descrevem a promoção da saúde como a qualificação de indivíduos e coletividades para atuar na melhoria da qualidade de vida, e a necessidade da intersetorialidade e interdisciplinaridade para a efetivação dessas ações ${ }^{22}$. Da mesma forma que na Inglaterra, as estratégias preventivas para doenças crônicas associadas à idade avançada têm-se tornado uma prioridade de saúde pública no Brasil23.

Esse cenário mostra a relevância de estimular a participação do usuário e da população, em conjunto com os profissionais, no sentido de construir a autonomia em relação aos determinantes de saúde. Nesse sentido, o aumento da qualidade das informações relacionadas com as DCNT, o empoderamento e a responsabilização dos sujeitos, assim como a promoção da saúde e a adoção de modos de vida saudáveis, são fundamentais para o enfrentamento dessas doenças ${ }^{24}$.

As equipes dos municípios entendem a forma de realização das reuniões como problema, sendo que o município B não mantém rotina de reuniões, e estas, quando ocorrem, têm como foco a resolução de problemas pontuais, não envolvendo toda a equipe. $\mathrm{O}$ trabalho em equipe, a partir das diretrizes do SUS, propõe a superação do modelo técnico hierarquizado e busca maior horizontalidade e flexibilidade dos diversos poderes, possibilitando autonomia e implicação dos envolvidos. Assim, o trabalho entre os profissionais que compõem a equipe de saúde pode ser considerado como uma rede de relações entre pessoas, poderes, saberes, afetos, interesses e desejos ${ }^{25}$. Dessa forma, a falta de integração entre os profissionais pode dificultar a mudança do modelo de atenção à saúde da população.

A inclusão dos ACS na equipe foi um avanço, no entanto, a prática da comunicação tradicional é recorrente, com a predominância de discursos verticalizados e uma escuta ainda limitada de outros segmentos sociais, o que representa uma das grandes dificuldades para mudanças na dinâmica das equipes e na participação da comunidade ${ }^{26}$, que pode ser evidenciado na fala a seguir: 
[...] a gente nunca tem oportunidade de reunir todo mundo, as agentes muito pouco... cada um resolve as suas [dificuldades] e acaba nem conversando com o colega [...]. (NB).

Reuniões normativas, com pouca participação da equipe, foram descritas por profissionais do município A. Os relatos denotam dificuldades para promover a integração da equipe na análise e planejamento de ações que respondam às demandas da comunidade. Uma pesquisa realizada no Distrito Federal, no Brasil, apontou que, nas reuniões de equipe, a transmissão de informações, geralmente já elaboradas por outros níveis hierárquicos, era a estratégia de comunicação predominante, reproduzindo formas tradicionais, verticalizadas e autoritárias de comunicação ${ }^{26}$.

... a gente geralmente acaba planejando sozinho e trazendo as coisas muito prontas. (EA).

Então a gente vinha aqui, às vezes tinha uns recados, aí tinha que ter um coordenador para trazer alguma coisa para o grupo, e não tinha muito foco. (ACSA).

Relações mais participativas tendem a propiciar o desenvolvimento de competências no trabalho dos profissionais, como maior autonomia e iniciativa, o que repercute em suas relações com a comunidade e cria possibilidades de compreensão de suas necessidades ${ }^{\mathbf{2 6}}$. O funcionamento dialógico e integrado torna o espaço coletivo, que discute casos clínicos, sanitários ou de gestão, e participa da vida da organização ${ }^{27}$.

Os profissionais da ESF têm atribuições comuns e específicas, conforme a Política Nacional de Atenção Básica ${ }^{15}$. Em meio às falas, emergiram conflitos ocasionados pelo desconhecimento das atribuições de cada profissão:

Tem colegas que não sabem o trabalho escondido que a gente faz, as incomodações, quando eles chegam 'já que tu não estás trabalhando'. (EB).
O sentimento de solidão e ausência de apoio no ambiente de trabalho foi outro aspecto evidenciado como uma das dificuldades vivenciadas pelas equipes:

Eu acho que às vezes liga no automático e trabalha, e a gente não tem com quem desabafar, porque a gente tem cada um suas funções, tem que dar conta de tudo [...]. (NB).

O AM oportuniza aos trabalhadores de saúde a discussão sobre o cotidiano e a organização do trabalho, buscando novas estratégias de atendimento e de ações de promoção à saúde ${ }^{\mathbf{2 8 , 2 9}}$. Nesse sentido, os apoiadores foram percebidos pelos profissionais da ESF como um reforço, na gestão municipal, para a necessidade de mudança nos processos de trabalho.

Um dos relatos revela um posicionamento que parece colocar população e profissionais em oposição de interesses:

Seria interessante criar uma visão de fora... porque a gente fala, e é a população contra os profissionais, é a população em primeiro lugar. (FIB).

Em meio à sensação de impotência para promover mudanças, os profissionais lembram do controle social como potencial aliado:

Teria que mostrar, ... principalmente para o Conselho Municipal de Saúde, ... só que ele é totalmente manipulado pela gestão, o pessoal senta, diz amém e deu. (FIB).

Esta fala revela o não empoderamento dos conselheiros para contrapor a gestão municipal. Outro estudo realizado em municípios do RS também revelou um controle social desarticulado e sem consciência de seu papel e responsabilidades, situação encontrada em vários municípios brasileiros ${ }^{30}$.

O cuidado na doença crônica apresenta características de permanência e longitudinalidade, exigindo, tanto do profissional quanto do usuário, persistência em relação às medidas de controle e tratamento, nem sempre fáceis 
de serem seguidas. Os profissionais manifestaram desconforto em relação a essa situação, entendendo que isso interfere no seu modo de cuidado.

A gente se acomoda um pouco. É sempre as mesmas coisas, tu não vês uma resposta, 'será que vale a pena investir?' ... e os pacientes crônicos acabam às vezes cansando a gente, ..., não evolui... (EB).

A sensação, por parte dos profissionais, de que as pessoas com DCNT são 'complicadas' cria um círculo vicioso que os desmotiva a investir nessas pessoas, impedindo a busca de meios inovadores e mais efetivos de educação em saúde. O núcleo desse problema é a dificuldade de criar vínculo, base para poder motivar o usuário ${ }^{19}$. Nesse sentido, as equipes revelaram uma certa acomodação e pouca reflexão sobre os processos de trabalho com o usuário com DCNT.

Eu acho que antes a gente tava fazendo os grupos de saúde sem muito pensar no que aquilo tava realmente trazendo de benefício [...] A gente acaba fazendo as coisas muito no automático e aí a gente não avalia o que fez. (DA).

Às vezes a gente fica aqui, não dá, a gente não consegue, ou faz sempre a mesma coisa e acha que não tem como fazer diferente. (EA).

Percebe-se que o cuidado é desenvolvido sem uma avaliação prévia sobre os modos de vida e o contexto dos usuários, o que promoveria maior sentido e implicação deles no seu cuidado. As diretrizes da Clínica Ampliada apontam para a implementação de mudanças nos processos de cuidado e na construção de projetos terapêuticos singulares. Ampliar a clínica implica colocar o foco da atenção no sujeito que está doente e que, também, apresenta outras necessidades. Nessa proposição, o trabalho em saúde deve estar voltado para as pessoas e coletivos, sem desconsiderar as singularidades; incorporar o subjetivo e o social, sem ignorar a doença; atuar em equipe, visando não apenas curar, reabilitar e prevenir, mas também promover a autonomia dos sujeitos e sua capacidade de reinventar e gerir a vida ${ }^{31}$.

A fragmentação do cuidado conforme o núcleo profissional foi evidenciada nos relatos:

Normalmente fazia uma visita num paciente focando a saúde bucal, ... via se tinha alguma cárie, um problema em alguma prótese (DA).

[...] a gente estava brincando hoje de manhã, esquartejamos ele. (EB).

Esse modo de organização dos processos de trabalho mostra as dificuldades dos profissionais em olhar para o sujeito e suas singularidades, e não apenas para a doença, a partir da sua área de conhecimento. Para implementar a integralidade do cuidado, é necessário aprofundar o debate sobre a natureza do processo de trabalho, sua micropolítica e importância na compreensão da organização da assistência à saúde 32 .

Os ACS participaram ativamente dos encontros do AM, sendo que os do município B entregaram uma carta para os apoiadores, destacando dois aspectos: a necessidade de serem capacitados para intervenções, principalmente, em saúde mental; e a diferença na forma como a população recebe os profissionais da equipe durante as visitas domiciliares.

Muitas vezes necessitaríamos acompanhamento com um profissional psicólogo, pois na nossa caminhada precisamos de ajuda diária, principalmente na área da saúde mental [...] muda muito quando nós entramos na casa de uma pessoa e quando a Dra. vai na casa, eles acolhem a ... de braços abertos,... mas quando nós vamos a situação é diferente. (ACSB).

Na visão dos ACS do município B, somente quem tem a experiência como agente de saúde, entrar na casa da pessoa, conhecer sua 
história, cultura, é que nos deixa face a face com a realidade, mudando a visão e a percepção da família.

Os relatos denotam que os ACS percebem diferenças de tratamento manifestado pelos usuários em relação às visitas realizadas pelo médico da equipe e por eles. Essa diferença pode estar relacionada com os efeitos da atenção à saúde centrada no modelo médico hegemônico que a população foi incorporando, que valoriza mais o saber médico e não reconhece o papel dos ACS. Estes são atores importantes que facilitam a comunicação entre o usuário e a equipe de ESF, aproximando-se das condições de saúde e de vida dos usuários, fortalecendo o vínculo entre os profissionais e a comunidade ${ }^{33}$.

Estudo sobre a implementação da governança clínica no sistema de saúde da Nova Zelândia mostrou que, embora os médicos sejam vistos como essenciais para o desenvolvimento da governança clínica, outros profissionais têm contribuições igualmente importantes para a melhoria efetiva da qualidade da assistência. O mesmo estudo reforça que isso requer trabalho em equipe e abordagens interprofissionais, pois os ambientes são complexos ${ }^{34}$.

\section{Qualificação do cuidado às pessoas com DCNT a partir do Apoio Matricial}

O cotidiano do trabalho em saúde pode levar à sensação de isolamento e à repetição de processos, sem muita reflexão sobre os resultados obtidos. O AM, na percepção dos profissionais, proporcionou escuta e problematização das situações vivenciadas pelas equipes.

Eu acho que está todo mundo meio que no automático [...] a gente fala 'aí que bom, é o dia da reunião' [do apoio], [... é um momento que a gente pode refletir, e as coisas dos casos faz a gente pensar sobre algumas coisas, [...], mas quem vem de fora dá um olhar diferente, [...] a gente acaba deixando para vocês um pouco do fardinho, [...] as angústias, alguém está nos escutando. (NB).

Semelhantemente a este estudo, outra pesquisa realizada em São Paulo mostrou que o AM fortalecia a construção de novas lógicas de trabalho por meio de trocas interdisciplinares e novas perspectivas de abordagem dos problemas pelas equipes ${ }^{7}$.

Havia uma expectativa inicial dos profissionais de que os pesquisadores trariam informações e palestras, no entanto, receberam bem a metodologia de trabalho, que rompeu com a transmissão vertical de conhecimento.

A gente achou que fosse assim, como nós iríamos agir, que vocês trouxessem os processos para nós. Não, chegaram aqui e fizeram a gente pensar! (ACSA).

A partir do AM, alguns trabalhadores sentiram-se empoderados para realizar mudanças ou, pelo menos, propô-las para os gestores.

Eu mudei algumas coisas, já tenho uma cartinha escrita que eu só estou esperando entrar o novo secretário, com minhas reivindicações. (NB).

Tivemos conquistas nesses encontros, tanto a gente reclamou, e conseguiu, nas sextas-feiras, uma hora de relax para pensar um pouco na gente. (EB).

Os momentos de encontro proporcionados pelo AM resultaram na avaliação das práticas, em processo baseado nos pressupostos da EPS. Estes pressupostos destacam a aprendizagem no trabalho, em que o aprender e o ensinar se incorporam ao cotidiano das organizações ${ }^{35}$.

A gente acabou sentando para conversar e avaliar os grupos e vendo que não tão sendo tão eficazes [...], aí a gente resolveu mudar isso. (DA).

A partir dessa avaliação, as equipes perceberam que é preciso realizar uma escuta ativa das necessidades dos usuários e planejar as ações a partir desta. 
Uma área não é igual a outra, ... a gente tem que ouvir o que a população quer. A gente não pode chegar com uma proposta pronta. (EA).

E aí chegou na hora e a XX [matriciadora] disse, não é assim, tu tens que ouvir o que eles querem, aí fui ouvir e mudou tudo. (ACSA).

Os relatos denotam que as atividades não contavam com a participação dos usuários em sua elaboração, eram ações verticalizadas, pois não foram construídas considerando as suas necessidades. A partir de uma dinâmica desenvolvida pelos apoiadores, denominada 'Saindo da Caixa', a qual propõe novas maneiras de pensar e agir, para além do olhar para a doença e da atitude prescritiva, foram emergindo novas estratégias de cuidado. As equipes de saúde relataram que a atividade os fez mudar práticas que se repetiam no cotidiano, e que os usuários foram receptivos a estas mudanças.

Novembro do ano passado nós fizemos a primeira saída da caixa, nos reunimos e fomos ao encontro da terceira idade e lá nós interagimos com eles, não fizemos palestra. (ACSA).

Estudo realizado nos Estados Unidos desenvolveu um instrumento de autoavaliação para ajudar as equipes de saúde a identificar e a direcionar os esforços de melhoria em seus programas educacionais de residência para cuidados crônicos. A maior melhoria foi observada incluindo o autogerenciamento do paciente como um componente central da prestação de cuidados de saúde e o uso de registros populacionais de doenças para monitorar a qualidade do atendimento prestado ${ }^{36}$.

O município B não fazia reuniões sistemáticas com toda a equipe, alegando muita demanda e falta de tempo. No entanto, a partir do AM, percebeu que, para que isso ocorra, é necessário o entendimento de que a discussão na equipe é prioridade e tem o potencial de qualificar as práticas.
E a gente vê que consegue se organizar, a gente diz falta de tempo, essa é a desculpa que tem no dia a dia. (EB).

O município A tem reuniões de equipe todas as quintas, mas, a partir do AM, percebeu que era necessário mudar a forma de organizar as reuniões.

As nossas reuniões também começaram a ter mais foco[...]. (MPA).

[...] a gente falava em linhas de cuidado e muita gente nem sabia o que era ..., a partir do que vocês trouxeram, a gente um dia fez um estudo sobre o que eram as linhas de cuidado, quais as linhas que a gente já tem, o que a gente podia aprimorar. (TEA).

Os profissionais foram convidados pela equipe de AM a analisarem suas atividades em relação às atribuições conforme a PNAB ${ }^{15}$. A partir dessa atividade, observou-se a sensibilização para a necessidade do trabalho interprofissional e o reconhecimento das atribuições dos outros profissionais.

A história de trabalhar a função de cada um, também eu achei importante porque, qual é o meu papel? [...] eu não consigo dar conta de tudo, [...] a gente não faz nem ideia do que ela faz na visita [referindo-se à ACS]. (NB).

A comunicação entre os ACS e os outros profissionais da equipe foi um aspecto que melhorou como consequência do processo de AM. Essa aproximação provocou mudanças no cuidado aos usuários, a partir do conhecimento do contexto destes, proporcionado pelos ACS.

... conheceram um pouco do trabalho delas, [...] nem fazia ideia que elas [ACS] têm conhecimento da medicação que eles tomam. (TEB).

... Muitas vezes tem um doente lá que precisa de exercício físico, mas ela (médica) não sabe onde ele mora, que condições ele vive, o que ele poderia fazer, daí fica difícil ela orientar uma coisa ... (ACSB). 
O AM pressupõe a cogestão e a construção compartilhada do cuidado, facilitando as relações comunicativas e dialógicas entre as organizações e os profissionais de saúde e entre estes e os usuários, oportunizando a participação ativa desses atores nos seus projetos terapêuticos ${ }^{37}$. A integralidade do cuidado emergiu na reflexão de um cirurgião-dentista durante o AM.

[...] aprendi a escutar... focar um pouco diferente, não só odontologia, não olho só a boca, fico realmente pensando o que mais que tem escondido por trás de tudo isso, [...]. (DA).

Assim, o AM amplia as possibilidades de realizar clínica ampliada e integração dialógica entre distintas profissões, já que se considera que, de modo isolado, não será possível assegurar uma abordagem integral ao paciente. O AM busca assegurar maior eficiência ao trabalho em saúde, mas também investir na construção de autonomia dos usuários. Sua utilização como instrumento concreto e cotidiano pressupõe certo grau de transformação do modo como se organizam e funcionam serviços de saúde 38 .

\section{Considerações finais}

$\mathrm{O}$ estudo revelou alguns entraves às equipes municipais para a efetivação da gestão e do cuidado às pessoas com DCNT. Os profissionais percebem essas dificuldades, mas não se sentem em condições de fazer o enfrentamento, colocando a gestão municipal como barreira. Verificou-se o descontentamento das equipes com o direcionamento assistencialista da gestão, que, na avaliação dos participantes, estimula o aumento de demandas apresentadas pela comunidade, com baixa implicação dos usuários e pouca resolutividade.

No entanto, para além da gestão, o que se observa é um trabalho no qual a dimensão interprofissional encontra-se reduzida, com um distanciamento entre os diversos saberes, que pouco se complementam. Os relatos e análises realizados apontam que, nos processos de trabalho na APS, ainda predomina um modelo de atenção prescritivo, focado na doença e com pouca atuação das equipes na atenção integral da saúde e no empoderamento dos usuários.

Considera-se que o AM se mostrou efetivo ao instigar as equipes de APS na análise dos respectivos processos de trabalho no cuidado às pessoas com DCNT. O AM também potencializou as relações comunicativas e dialógicas entre os participantes, ampliando a cogestão dos processos de cuidado. Ou seja, a partir dos encontros de AM, as equipes reconheceram a necessidade de mudança nas intervenções individuais e coletivas e realizaram algumas ações de planejamento e cuidado mais horizontalizados e interdisciplinares, incluindo os usuários. A continuidade dessas mudanças é que poderão ampliar e qualificar os resultados no cuidado às pessoas com DCNT.

Por se tratar de AM realizado por pesquisadores, uma das limitações do estudo é a dificuldade de acompanhamento dos efeitos em longo prazo após a finalização da pesquisa. Sugerem-se investigações semelhantes a serem realizadas em outros locais para que possam reforçar e complementar os resultados deste estudo.

\section{Colaboradoras}

Medeiros CRG (0000-0001-9466-0437)*, Freitag AL (0000-0002-4858-4389)*, Martinez LSE (0000-0002-1301-3885)*, Saldanha OMFL (0000-0003-4189-4779)*, Grave MQ (0000-0001-7185-727X)*, Jager LK (0000-0003-2816-2231)* e Dhein G (00000002-9379-6479)* contribuíram para a concepção do estudo, coleta e análise dos dados, elaboração e revisão do artigo.
*Orcid (Open Researcher and Contributor ID). 


\section{Referências}

1. World Health Organization. World Health Statistic 2017: monitoring health for the SDGs, Sustainable Development Goals. Geneva: WHO; 2017.

2. Malta DC, França E, Abreu DMX, et al. Mortality due to noncommunicable diseases in Brazil, 1990 to 2015, according to estimates from the Global Burden of Disease study. São Paulo Med J. 2017; 135(3):213-21.

3. Brasil. Ministério da Saúde. Portaria n ${ }^{\circ} 252$, de 19 de fevereiro 2013. (Revogada pela PRT GM/MS nº 483 de 01/04/2014). Institui a Rede de Atenção à Saúde das Pessoas com Doenças Crônicas no âmbito do Sistema Único de Saúde (SUS) [internet]. Diário Oficial da União. 20 Fev 2013. [acesso em 2020 mar 30]. Disponível em: http://bvsms.saude.gov.br/bvs/saudelegis/gm/2013/prt0252_19_02_2013.html.

4. Brasil. Ministério da Saúde. Portaria $n^{\circ} 483$, de $1^{\circ}$ de abril de 2014. Redefine a Rede de Atenção à Saúde das Pessoas com Doenças Crônicas no âmbito do Sistema Único de Saúde (SUS) e estabelece diretrizes para a organização das suas linhas de cuidado [internet]. Diário Oficial da União. 2 Abr 2014. [acesso em 2020 mar 30]. Disponível em: http://bvsms.saude.gov.br/ bvs/saudelegis/gm/2014/prt0483_01_04_2014.html.

5. Raupp LM, Dhein G, Medeiros CRG, et al. Doenças crônicas e trajetórias assistenciais: avaliação do sistema de saúde de pequenos municípios. Physis. 2015; 25(2):615-34.

6. Castro CP, Oliveira MM, Campos GWS. Apoio Matricial no SUS Campinas: análise da consolidação de uma prática interprofissional na rede de saúde. $\mathrm{Ci}$ ênc. Saúde Colet. 2016; 21(6):1625-36.

7. Barros JO, Gonçalves RMA, Kaltner RM, et al. Estratégia do apoio matricial: a experiência de duas equipes do Núcleo de Apoio à Saúde da Família (NASF) da cidade de São Paulo, Brasil. Ciênc. Saúde Colet. 2015; 20(9):2847-2856.

8. Cardoso AS, Nascimento MC. Comunicação no Pro- grama Saúde da Família: o agente de saúde como elo integrador entre a equipe e a comunidade. Ciênc. Saúde Coletiva. 2010; 15(supl1):1509-1520.

9. Hirdes A. A perspectiva dos profissionais da Atenção Primária à Saúde sobre o apoio matricial em saúde mental. Ciênc. Saúde Colet. 2010; 20(2):371-382.

10. Jorge MS, Sousa FSP, Franco TB. Apoio matricial: dispositivo para resolução de casos clínicos de saúde mental na Atenção Primária à Saúde. Rev Bras Enferm. 2013; 66(5):38-44.

11. Brasil. Ministério da Saúde. Agenda nacional de prioridades de pesquisa em saúde. 2. ed. Brasília, DF: Ministério da Saúde; 2008.

12. Baremblitt GF. Compêndio de análise institucional e outras correntes: teoria e prática 6. ed. Belo Horizonte: IFG; 2012.

13. Ponte HMS, Oliveira LCO, Ávila M. Desafios da operacionalização do Método da Roda: experiência em Sobral (CE). Saúde debate. 2016; 40(108):34-47.

14. Bardin L. Análise de conteúdo. São Paulo: Edições $70 ; 2011$.

15. Brasil. Ministério da Saúde. Portaria no 2.436 , de 21 de setembro de 2017. Aprova a Política Nacional de Atenção Básica, estabelecendo a revisão das diretrizes para a organização da Atenção Básica, no âmbito do Sistema Único de Saúde (SUS) [internet]. Diário Oficial da União. 22 Set 2017. [acesso em 2020 mar 30]. Disponível em: http://www.saude.df.gov.br/wp-conteudo/uploads/2018/04/Portaria-n\%C2\%BA-2436-2017-Minist\%C3\%A9rio-da-Sa\%C3\%BAde-Aprova-a-Pol\%C3\%ADtica-Nacional-de-Aten\%C3\%A7\%C3\%A3o-B\%C3\%Alsica..pdf.

16. Brasil. Ministério da Saúde. Portaria $n^{\circ} 1.996$, de 20 de agosto de 2007. Dispõe sobre as diretrizes para a implementação da política nacional de educação permanente em saúde [internet]. Diário Oficial da 
União. 22 Ago 2007. [acesso em 2020 mar 30]. Disponível em: http://bvsms.saude.gov.br/bvs/saudelegis/gm/2007/prt1996_20_08_2007.html.

17. Brasil. Ministério da Saúde. Resolução $n^{\circ} 466$, de 12 de dezembro de 2012. Sobre pesquisas envolvendo seres humanos [internet]. Diário Oficial da União. 12 Dez 2012. [acesso em 2020 mar 30]. Disponível em: https://bvsms.saude.gov.br/bvs/saudelegis/cns/2013/ res0466_12_12_2012.html.

18. Frutoso MFP, Mendes R, Rosa KRM, et al. Gestão local de saúde em território de vulnerabilidade: motivações e racionalidades. Saúde debate. 2015; 39(105):337349.

19. Silocchi C, Jungues JR. Equipes de Atenção Primária: dificuldades no cuidado de pessoas com Doenças Crônicas Não Transmissíveis. Trab. Educ. Saúde. 2017; 15(2):599-615.

20. Bonomi A, Wagner EH, Glasgow RE, et al. Assessment of Chronic Illness Care (ACIC): A Practical Tool to Measure Quality Improvement. Health Serv Res. 2002; 37(3):791-820, 2002.

21. Figueiredo EN. Estratégia Saúde da Família e Núcleo de Apoio à Saúde da Família: diretrizes e fundamentos. São Paulo: UNIFESP; 2011.

22. Heidemann ITSB, Boehs AE, Fernandes GCM, et al. Promoção da saúde e qualidade de vida: concepções da carta de Ottawa em produção científica. Ciênc. Cuid Saúde. 2012; 11(3):613-19.

23. Liljas AEM, Walters K, Jovicic A, et al. Strategies to improve engagement of 'hard to reach' older people in research on health promotion: a systematic review. BMC Public Health. 2017; 17(1):349.

24. Mássimo EAL, Souza HNF, Freitas MIF. Doenças crônicas não transmissíveis, risco e promoção da saúde: construções sociais de participantes do Vigitel. Ciênc. Saúde Colet. 2015; 20(3):679-88.

25. Fortuna CM, Midhima SM, Matumoto S, et al. O tra- balho de equipe no programa saúde da família: reflexões a partir de conceitos do processo grupal e de grupos operativos. Rev Latino-Am Enfermagem. 2005; 13(2):262-268.

26. Cardoso JR, Oliveira GN, Furlan PG. Gestão democrática e práticas de apoio institucional na Atenção Primária à Saúde no Distrito Federal, Brasil. Cad. Saúde Pública [internet]. 2016; 32(3): e00009315. [acesso em 2020 mar 30]. Disponível em: http://www.scielo. br/pdf/csp/v32n3/0102-311X-csp-32-03-e00009315. pdf.

27. Campos GWS, Domitti AC. Apoio matricial e equipe de referência: uma metodologia para gestão do trabalho interdisciplinar em saúde. Cad. Saúde Pública. 2008 ; 14:863-70.

28. Borelli M, Domene SMA, Mais LA, et al. A inserção do nutricionista na Atenção Básica: uma proposta para o matriciamento da atenção nutricional. Ciênc. Saúde Colet. 2015; 20(9):2765-2778.

29. Campos RTO, Campos GWS, Ferrer AL, et al. Avaliação de estratégias inovadoras na organização da Atenção Primária à Saúde. Rev. Saúde Pública. 2012; 46(1):43-50.

30. Medeiros CRG. Rede de cuidado em saúde: o dilema dos pequenos municípios [tese]. Porto Alegre: Universidade Federal do Rio Grande do Sul; 2013. 201 p.

31. Campos GWS, Cunha GT, Figueiredo MD. Práxis e Formação Paideia: apoio e cogestão em saúde. São Paulo: Hucitec; 2013.

32. Malta DC, Merhy EE. The path of the line of care from the perspective of non-transmissible chronic diseases. Interface. 2010; 14(34):593-605.

33. Santos CW, Filho MCF. Agentes Comunitários de Saúde: uma perspectiva do capital social. Ciênc. Saúde Colet. 2016; 21(5):1659-1667.

34. Galud R, Horsbugh S. Are some health professionals more cognizant of clinical governance development 
concepts than others? Findings from a New Zealand study. J Public Health. 2015; 38(2):363-370.

35. Mancia J, Cabral LC, Koerich MS. Educação permanente no contexto da enfermagem e na saúde. Rev. Bras. Enferm.2004; 57(5):605-610.

36. Bowen JL, Provost L, Stevens DP, et al. Avaliação da Educação para a Assistência à Doença Crônica (ACIC-E): uma ferramenta para rastrear o re-design educacional para melhorar a educação em cuidados crônicos. J Gen Intern Med. 2010; 25(4):593-609.
37. Martins MM, Campos GWS. Apoios matricial e institucional: analisando suas construções. Ciênc. Saúde Colet. 2015; 20(1):229-238.

38. Saupe R, Cutolo LRA. Wendhausen ALP. Competências dos profissionais da saúde para o trabalho interdisciplinar. Interface. 2005; 9:521-36.

Recebido em 01/08/2019

Aprovado em 10/03/2020

Conflito de interesses: inexistente

Suporte financeiro: não houve 